\title{
New delay-dependent synchronization criteria for uncertain Lur'e systems via time-varying delayed feedback control
}

\author{
Yanmeng Wang ${ }^{\mathrm{a}}$, Lianglin Xiong ${ }^{\mathrm{b}, *}$, Xinzhi Liu ${ }^{\mathrm{c}}$, Haiyang Zhang ${ }^{\mathrm{d}}$ \\ ${ }^{a}$ College of Sciences, Nanjing University of Aeronautics and Astronautics, 210016 Nanjing, China. \\ ${ }^{b}$ School of Mathematical Sciences, Yunnan Minzu University, 650500 Kunming, China. \\ ${ }^{c}$ Department of Applied Mathematics, University of Waterloo Waterloo, Ontario, Canada N2L 3G1. \\ ${ }^{d}$ School of Science, Nanjing University of Science and Technology, 210094 Nanjing, China.
}

Communicated by R. Saadati

\begin{abstract}
This paper studies the problem of master-slave synchronization for uncertain Lur'e system via time-varying delayed feedback control. It proves a new inequality involving double integrals, which can reduce the conservatism of the known Jensen's like inequalities according to our analysis. By employing this new inequality and a new class of novel mode-dependent augmented Lyapunov-Krasovskii functional (LKF), it establishes some novel synchronization criteria, where the controller gain can be achieved by solving a set of linear matrix inequalities (LMIs). Two examples with numerical simulations are given to illustrate the feasibility and the superiority of our methods. (C)2017 All rights reserved.
\end{abstract}

Keywords: Uncertain Lur'e system, synchronization, improved integral inequality, linear matrix inequalities. 2010 MSC: 47H10, 54H25.

\section{Introduction}

During the last two decades, chaos synchronization has received much attention due to its theoretical importance and practical applications, see for example $[1-4,6,9,11,12,15,17,18,22]$ and references therein. Such synchronization has been widely explored in a variety of fields including physical, chemical and ecological systems, human heartbeat regulation, secure communications, and so on. Moreover, a number of master-slave synchronization schemes for Lur'e systems have been proposed $[3,4,12,15,17$, 18].

Recently, the effect of delay on synchronization between two chaotic systems has been reported in many literatures due to the propagation delay frequently encountered in remote master-slave synchronization scheme. Guo and Zhong in [6] and Yalcin et al. in [21] derived some delay-independent [9] and delay-dependent synchronization criteria for global asymptotic stability of the error system, which

\footnotetext{
${ }^{*}$ Corresponding author

Email addresses: 18213498367@163.com (Yanmeng Wang), lianglin_5318@163.com (Lianglin Xiong), xinzhi.liu@uwaterloo.ca (Xinzhi Liu), Haiya287@126.com (Haiyang Zhang)
}

doi:10.22436/jnsa.010.04.52 
are expressed as LMIs and derived from extended LKFs. Liao and Chen [11] and Cao et al. [1] further generalized and improved the results in [21] and employed model transformation, which leads to some conservative synchronization criteria for inducing additional terms. Shortly, some new approaches are employed to avoid using model transformation and derive much less conservative synchronization conditions, for example, Xiang et al. [20], He et al. [8] used integral inequality [22] in the derivative of Lyapunov functional respectively, and it turned out that the permissible delay threshold can be fairly enlarged both in theory and in numerical experiment. However, with the development of the time, many methods to reduce the conservatism have been produced and applied in many other fields such as the integral inequality techniques used in neural network [19], and achieved good results. It should be pointed out that, most of the above articles are discussed only in constant delay. In practice, the time-varying delay often arises and may vary in a range.

Motivated by the above discussions, this paper investigates the problem of delay-dependent synchronization for uncertain Lur'e system with time-varying delayed feedback control. Before the problem of synchronization of uncertain Lur'e systems is investigated, this paper introduced a class of new tripleintegral inequality used in the following LKFs to get less conservative criteria. The LKF contains not only double-integral terms but also triple-integral terms. And using some other effective techniques, such as a piecewise analysis method and the general free-weighting matrix method, some sufficient conditions on the existence of a delayed error feedback controller derived in the form of LMIs are less conservative than the existing results. Two examples with numerical simulations are given to illustrate the effectiveness and superiority of the obtained criteria.

Notation: $R^{n}$ denotes the $n$-dimensional Euclidean space, and $R^{n \times m}$ denotes the set of all $n \times m$ real matrices. $\mathrm{P} \in \mathrm{R}^{\mathrm{n} \times n}$ and $\mathrm{P}>0$ (respectively, $\mathrm{P}<0$ ) show that $\mathrm{P}$ is a positive (respectively, negative) definite matrix. $\operatorname{diag}\left\{a_{1}, a_{2}, \ldots, a_{n}\right\}$ represents a diagonal matrix with diagonal elements $a_{1}, a_{2}, \ldots, a_{n}$.* denotes a symmetric term in a symmetric matrix.

\section{Problem statement and preliminaries}

Consider the following master-slave synchronization scheme using time-varying delayed feedback control:

$$
\begin{aligned}
& M:\left\{\begin{aligned}
\dot{x}(t) & =(A+\Delta A(t)) x(t)+(B+\Delta B(t)) \varphi(C x(t)), \\
p(t) & =H x(t),
\end{aligned}\right. \\
& S:\left\{\begin{aligned}
\dot{y}(t) & =(A+\Delta A(t)) y(t)+(B+\Delta B(t)) \varphi(C y(t))+u(t), \\
q(t) & =H y(t), \\
& C: u(t)=M(p(t-h(t))-q(t-h(t))),
\end{aligned}\right.
\end{aligned}
$$

with master system $M$, slave system $S$ and controller $C$, where the time-delay $0 \leqslant h(t) \leqslant h_{M}$ and $0 \leqslant \dot{h}(t) \leqslant h_{d}<1$. The master and slave systems are Lur'e systems with state vectors $x, y \in R^{n}$, and the output vectors $p, q \in R^{l}$, respectively. The matrices $A \in R^{n \times n}, B \in R^{n \times m}, C \in R^{m \times n}$ and $H \in R^{l \times n}$ are known constant matrices. The nonlinearity $\varphi(\cdot)$ is time-invariant, decoupled, and satisfies a sector condition with $\varphi_{i}(\sigma)(i=1,2, \ldots, m)$ belonging to a sector $[0, k]$, i.e.,

$$
\varphi_{i}(\sigma)\left(\varphi_{i}(\sigma)-k \sigma\right) \leqslant 0(\forall t \geqslant 0, \forall \sigma \in R) .
$$

$\Delta \mathrm{A}(\mathrm{t})$ and $\Delta \mathrm{B}(\mathrm{t})$ are time-varying uncertain matrices of appropriate dimensions, which are assumed to be of the following form:

$$
\left[\begin{array}{ll}
\Delta \mathrm{A}(\mathrm{t}) & \Delta \mathrm{B}(\mathrm{t})
\end{array}\right]=\mathrm{NF}(\mathrm{t})\left[\begin{array}{ll}
E_{\mathrm{a}} & \mathrm{E}_{\mathrm{b}}
\end{array}\right],
$$

where $N, E_{a}$ and $E_{b}$ are known real constant matrices of appropriate dimensions and $F(t)$ is a timevarying uncertain matrix satisfying

$$
F(t)^{T} F(t) \leqslant I, \quad \forall t \geqslant 0
$$

Defining a signal $e(t)=x(t)-y(t)$ as the synchronization error, we have the uncertain error dynamical system in the form: 


$$
\dot{e}(t)=(A+\Delta A(t)) e(t)+(B+\Delta B(t)) \eta(C e(t), y(t))-M H e(t-h(t)),
$$

where $\eta(\mathrm{Ce}, \mathrm{y})=\varphi(\mathrm{Ce}+\mathrm{Cy})-\varphi(\mathrm{C} y)$. Let $\mathrm{C}=\left[\mathrm{c}_{1}, \mathrm{c}_{2}, \ldots, \mathrm{c}_{\mathrm{m}}\right]^{\top}, \mathrm{c}_{\mathrm{i}} \in \mathrm{R}^{n}, i=1,2, \ldots, \mathrm{m}$. The nonlinearity $\eta(\mathrm{Ce}, \mathrm{y})$ is assumed to belong to the sector $[0, k]$, i.e., for all $t \geqslant 0$, and for all $e, y$,

$$
\eta_{i}\left(c_{i} e, y\right)\left(\eta_{i}\left(c_{i} e, y\right)-k c_{i} e\right) \leqslant 0
$$

By using (2.3), the uncertain error dynamical system (2.4) can be written as follows:

$$
\dot{e}(t)=A e(t)+B \eta(C e(t), y(t))-M H e(t-h(t))+N P(t),
$$

where

$$
\begin{aligned}
& P(t)=F(t) \tau \xi(t), \quad \tau=\left[\begin{array}{lllllllllll}
E_{a} & 0 & 0 & 0 & E_{b} & 0 & 0 & 0 & 0 & 0 & 0
\end{array}\right], \\
& \xi^{\top}(t)=\left[\begin{array}{llllll}
e^{\top}(t) & e^{\top}\left(t-\frac{h(t)}{2}\right) & e^{\top}(t-h(t)) & \dot{e}^{\top}(t) & \eta^{\top}(t) & \varphi^{\top}(t)
\end{array}\right. \\
& \frac{1}{h(t)} \int_{t-\frac{h(t)}{2}}^{t} e^{T}(s) d s \quad \frac{1}{h(t)} \int_{t-h(t)}^{t} e^{T}(s) d s \\
& \left.\frac{1}{h^{2}(t)} \int_{-\frac{h(t)}{2}}^{0} \int_{t+\theta}^{t} e^{T}(s) d s d \theta \quad \frac{1}{h^{2}(t)} \int_{-h(t)}^{0} \int_{t+\theta}^{t} e^{T}(s) d s d \theta \quad P^{\top}(t)\right] \text {. }
\end{aligned}
$$

The purpose of this paper is to study the mater-slave synchronization for uncertain Lur'e systems and design the controller (2.1), i.e., to find the controller gain $M$, such that the system described by (2.5) and (2.6) is robustly asymptotically stable, which means that the master system and the slave system are synchronized. The following lemmas are used in deriving synchronization criteria.

Lemma $2.1([13,14])$. For a given matrix $\mathrm{R}>0$, any differential function $\omega:[a, b] \rightarrow R^{n}$, the following inequality holds:

$$
\begin{aligned}
& \int_{a}^{b} \dot{\omega}^{\top}(u) R \dot{\omega}(u) d u \geqslant \frac{1}{b-a}(\omega(b)-\omega(a))^{\top} R(\omega(b)-\omega(a))+\frac{3}{b-a} \Omega_{1}^{\top} R \Omega_{1}, \\
& \int_{a}^{b} \omega^{\top}(u) R \omega(u) d u \geqslant \frac{1}{b-a}\left(\int_{a}^{b} \omega(u) d u\right)^{\top} R\left(\int_{a}^{b} \omega(u) d u\right)+\frac{3}{b-a} \Omega_{2}^{\top} R \Omega_{2},
\end{aligned}
$$

where

$$
\begin{aligned}
& \Omega_{1}=\omega(b)+\omega(a)-\frac{2}{b-a} \int_{a}^{b} \omega(u) d u, \\
& \Omega_{2}=\int_{a}^{b} \omega(s) d s-\frac{2}{b-a} \int_{a}^{b} \int_{a}^{s} \omega(r) d r d s .
\end{aligned}
$$

Lemma 2.2. For a given matrix $\mathrm{R}>0$, the following inequality holds for all continuously differentiable function $\omega \in[t-h, t] \rightarrow R^{n}$ :

$$
\int_{-h}^{0} \int_{t+\theta}^{t} \dot{\omega}^{\top}(s) R \dot{\omega}(s) d s d \theta \geqslant 6 \Omega_{3}^{\top}\left(\begin{array}{ccc}
R & R & -4 R \\
* & 3 R & -8 R \\
* & * & 24 R
\end{array}\right) \Omega_{3}
$$

where

$$
\Omega_{3}^{T}=\left(\omega^{\top}(t) \quad \frac{1}{h} \int_{t-h}^{t} \omega^{\top}(s) d s \quad \frac{1}{h^{2}} \int_{-h}^{0} \int_{t+\theta}^{t} \omega^{T}(s) d s d \theta\right) .
$$

Proof. For any continuous function $\omega(t) \in[t-h, t]$ and which admits a continuous derivative, define the function $z$ by

$$
z(s)=\dot{w}(s)-\frac{2}{h^{2}} A+\frac{4 g(s)}{h^{3}} B, s \in[t-h, t],
$$


where

$$
\begin{aligned}
A & =h \omega(t)-\int_{t-h}^{t} \omega(s) d s, \\
B & =h \omega(t)+2 \int_{t-h}^{t} \omega(s) d s-\frac{6}{h} \int_{-h}^{0} \int_{t+\theta}^{t} \omega(s) d s d \theta, \\
g(s) & =3 t-h-3 s .
\end{aligned}
$$

The computation of $\int_{-\mathrm{h}}^{0} \int_{\mathrm{t}+\theta}^{\mathrm{t}} z^{\mathrm{T}}(\mathrm{s}) \mathrm{Rz}(\mathrm{s}) \mathrm{dsd} \theta$ leads to

$$
\begin{aligned}
\int_{-h}^{0} \int_{t+\theta}^{t} z^{\top}(s) R z(s) d s d \theta= & \int_{-h}^{0} \int_{t+\theta}^{t} \dot{\omega}^{\top}(s) R \dot{\omega}(s) d s d \theta-\frac{4}{h^{2}} \int_{-h}^{0} \int_{t+\theta}^{t} \dot{\omega}^{\top}(s) d s d \theta R A \\
& +\frac{4}{h^{4}} \int_{-h}^{0} \int_{t+\theta}^{t} d s d \theta A^{\top} R A-\frac{16}{h^{5}} \int_{-h}^{0} \int_{t+\theta}^{t} g(s) d s d \theta A^{\top} R B \\
& +\frac{16}{h^{6}} \int_{-h}^{0} \int_{t+\theta}^{t} g^{2}(s) d s d \theta B^{\top} R B+\frac{8}{h^{3}} \int_{-h}^{0} \int_{t+\theta}^{t} g(s) \dot{w}(s) d s d \theta R B .
\end{aligned}
$$

Simple calculation ensures that

$$
\begin{gathered}
\int_{-h}^{0} \int_{t+\theta}^{t} \dot{\omega}(s) d s d \theta=A, \\
\int_{-h}^{0} \int_{t+\theta}^{t} d s d \theta=\frac{1}{2} h^{2}, \\
\int_{-h}^{0} \int_{t+\theta}^{t} g(s) d s d \theta=0, \\
\int_{-h}^{0} \int_{t+\theta}^{t} g^{2}(s) d s d \theta=\frac{1}{4} h^{4},
\end{gathered}
$$

and integration by parts ensures that

$$
\int_{-h}^{0} \int_{t+\theta}^{t} g(s) \dot{\omega}(s) d s d \theta=-h B
$$

It thus follows from $R>0$ that

$$
\int_{-h}^{0} \int_{t+\theta}^{t} z^{\top}(s) R z(s) d s d \theta=\int_{-h}^{0} \int_{t+\theta}^{t} \dot{\omega}^{\top}(s) R \dot{\omega}(s) d s d \theta-\frac{2}{h^{2}} A^{\top} R A-\frac{4}{h^{2}} B^{\top} R B \geqslant 0 .
$$

More specifically, one can see

$$
\int_{-h}^{0} \int_{t+\theta}^{t} \dot{\omega}^{\top}(s) R \dot{\omega}(s) d s d \theta \geqslant \frac{2}{h^{2}}\left(A^{\top} R A+2 B^{\top} R B\right),
$$

or equivalently

$$
\int_{-h}^{0} \int_{t+\theta}^{t} \dot{\omega}^{\top}(s) R \dot{\omega}(s) d s d \theta \geqslant \Omega_{3}^{\top}\left(\begin{array}{ccc}
6 R & 6 R & -24 R \\
* & 18 R & -48 R \\
* & * & 144 R
\end{array}\right) \Omega_{3},
$$

with $\Omega_{3}^{T}=\left(\omega^{\top}(t) \quad \frac{1}{h} \int_{t-h}^{t} \omega^{\top}(s) d s \quad \frac{1}{h^{2}} \int_{-h}^{0} \int_{t+\theta}^{t} \omega^{\top}(s) d s d \theta\right)$. This completes the proof.

Remark 2.3. It is worth mentioning that the choice of function $z(\mathrm{~s})$ is essential in the proof of Lemma 2.2. On the one hand, the function $g(s)$ should be constructed to make $\int_{-h}^{0} \int_{t+\theta}^{t} g(s) d s d \theta A^{\top} R B=0$, and lead 
to the right-hand of (2.7) in a standard quadratic form. Therefore, according to the computation for the integration, we choose $g(s)=3 t-h-3 s$. On the other hand, to unify the coefficient $\frac{1}{h^{2}}$ of the expression (2.7), $z(\mathrm{~s})$ should contain the coefficient $\frac{1}{\mathrm{~h}^{3}}$.

Remark 2.4. It can be seen that the inequality in [16, Lemma 1] is equivalent to the following one

$$
\int_{-h}^{0} \int_{t+\theta}^{t} \dot{\omega}^{\top}(s) R \dot{\omega}(s) d s d \theta \geqslant \frac{2}{h^{2}} A^{\top} R A,
$$

with $A$ defined in Lemma 2.2. Clearly, inequality (2.7) gives a better estimation than the inequality in [16, Lemma 1] does. Hence, Lemma 2.2 is less conservative than [16, Lemma 1]. It is worthy to note that this improvement is allowed by using an extra signal $\int_{-\mathrm{h}}^{0} \int_{\mathrm{t}+\theta}^{\mathrm{t}} \omega(\mathrm{s}) \mathrm{dsd} \theta$, not only the signals $\omega(\mathrm{s})$ and $\int_{t-h}^{t} \omega(s) d s$. Since [16, Lemma 1] has been widely applied in stability analysis of time-delay systems, Lemma 2.2 will be very important and applicable to analyze the delay-dependent stabilization for many kinds of delay dynamical systems and provide less conservative stability results.

\section{Main results}

We will establish a new delay-dependent stability criterion for the master-slave system by using the new inequality proposed above Lemma 2.2, which gives some less conservative sufficient conditions. Now we are in the position to state and prove the main results.

Theorem 3.1. The error system (2.6) satisfies the condition (2.5) is robustly asymptotically stable for given values $\mathrm{h}(\mathrm{t})$ in $\left\{0, \mathrm{~h}_{\mathrm{M}}\right\}$ and $\dot{\mathrm{h}}(\mathrm{t})$ in $\left\{0, \mathrm{~h}_{\mathrm{d}}\right\}$ and $\mathrm{q}>0$, if there exist appropriate dimensional matrices $\mathrm{L}_{1}, \mathrm{~L}_{2}$, and positive diagonal matrices $\Lambda=\operatorname{diag}\left\{\lambda_{1}, \lambda_{2}, \ldots \lambda_{m}\right\}>0, T_{i}=\operatorname{diag}\left\{t_{i 1}, t_{i 2}, \ldots, t_{i m}\right\}>0,(i=1,2)$, and positive matrices $\mathrm{P}=\left[\mathrm{P}_{\mathrm{ij}}\right]_{5 \times 5}, \mathrm{Q}_{\mathrm{i}}(\mathrm{i}=1,2, \ldots, 8)$ such that the following LMI holds:

$$
\Xi=\left[\begin{array}{cc}
E & 0 \\
0 & -q I
\end{array}\right]+L X+X^{\top} L^{\top}+q \tau^{\top} \tau<0,
$$

where

$$
\begin{aligned}
& E=\left[E_{i j}\right]_{10 \times 10}, \quad E_{i j}=E_{j i}, \quad i, j=1,2, \cdots, 10, \\
& E_{11}= P_{12}+P_{13}+\frac{h}{2} P_{14}+h P_{15}+P_{12}^{\top}+P_{13}^{\top}+\frac{h}{2} P_{14}^{\top}+h P_{15}^{\top}+Q_{1}+Q_{2}-\frac{4(2-\dot{h})}{h} Q_{3} \\
&-\frac{4(1-\dot{h})}{h} Q_{4}+\frac{h}{2} Q_{5}+h Q_{6}-3(2-\dot{h}) Q_{7}-6(1-\dot{h}) Q_{8}, \\
& E_{12}=-\left(1-\frac{\dot{h}}{2}\right) P_{12}-\frac{2(2-\dot{h})}{h} Q_{3} \\
& E_{13}=-(1-\dot{h}) P_{13}-\frac{2(1-\dot{h})}{h} Q_{4}, \\
& E_{14}= P_{11}, \\
& E_{15}= k C^{\top} T_{1}, \\
& E_{16}= k C^{\top} T_{2}, \\
& E_{17}=-\left(1-\frac{\dot{h}}{2}\right) h P_{14}+h P_{22}+h P_{23}^{\top}+\frac{1}{2} h^{2} P_{24}^{\top}+h^{2} P_{25}^{\top}+\frac{12(2-\dot{h})}{h{ }_{M}} Q_{3}-6(2-\dot{h}) Q_{7}, \\
& E_{18}=-(1-\dot{h}) h P_{15}+h P_{23}+h P_{33}+\frac{1}{2} h^{2} P_{34}^{\top}+h^{2} P_{35}^{\top}+\frac{6(1-\dot{h})}{h} Q_{4}-6(1-\dot{h}) Q_{8}, \\
& E_{19}= h^{2} P_{24}+h^{2} P_{34}+\frac{1}{2} h^{3} P_{44}+h^{3} P_{45}^{\top}+48(2-\dot{h}) Q_{7},
\end{aligned}
$$




$$
\begin{aligned}
& E_{110}=h^{2} P_{25}+h^{2} P_{35}+\frac{1}{2} h^{3} P_{45}+h^{3} P_{55}+24(1-\dot{h}) Q_{8}, \\
& \mathrm{E}_{22}=-\left(1-\frac{\dot{\mathrm{h}}}{2}\right) \mathrm{Q}_{1}-\frac{4(2-\dot{\mathrm{h}})}{\mathrm{h}_{\mathrm{M}}} \mathrm{Q}_{3}, \\
& E_{27}=-\left(1-\frac{\dot{h}}{2}\right) h P_{22}+\frac{12(2-\dot{h})}{h_{M}} Q_{3} \text {, } \\
& \mathrm{E}_{28}=-\left(1-\frac{\dot{\mathrm{h}}}{2}\right) \mathrm{hP}_{23} \text {, } \\
& \mathrm{E}_{29}=-\left(1-\frac{\dot{\mathrm{h}}}{2}\right) \mathrm{h}^{2} \mathrm{P}_{24} \\
& \mathrm{E}_{210}=-\left(1-\frac{\dot{\mathrm{h}}}{2}\right) \mathrm{h}^{2} \mathrm{P}_{25} \text {, } \\
& E_{33}=-(1-\dot{h}) Q_{2}-\frac{4(1-\dot{h})}{h_{M}} Q_{4} \\
& \mathrm{E}_{37}=-(1-\dot{\mathrm{h}}) h \mathrm{P}_{23}^{\top} \text {, } \\
& E_{38}=-(1-\dot{h}) h P_{33}+\frac{6(1-\dot{h})}{h_{M}} Q_{4} \text {, } \\
& \mathrm{E}_{39}=-(1-\dot{\mathrm{h}}) \mathrm{h}^{2} \mathrm{P}_{34} \text {, } \\
& \mathrm{E}_{310}=-(1-\dot{\mathrm{h}}) \mathrm{h}^{2} \mathrm{P}_{35} \text {, } \\
& \mathrm{E}_{44}=\frac{\mathrm{h}}{2} \mathrm{Q}_{3}+\mathrm{h} \mathrm{Q}_{4}+\frac{1}{8} \mathrm{~h}^{2} \mathrm{Q}_{7}+\frac{1}{2} \mathrm{~h}^{2} \mathrm{Q}_{8}, \\
& \mathrm{E}_{46}=\wedge \mathrm{C} \text {, } \\
& \mathrm{E}_{47}=\mathrm{hP}_{12} \text {, } \\
& \mathrm{E}_{48}=\mathrm{hP}_{13} \text {, } \\
& \mathrm{E}_{49}=\mathrm{h}^{2} \mathrm{P}_{14} \\
& \mathrm{E}_{410}=\mathrm{h}^{2} \mathrm{P}_{15} \text {, } \\
& E_{55}=-2 T_{1} \text {, } \\
& \mathrm{E}_{66}=-2 \mathrm{~T}_{2} \text {, } \\
& \mathrm{E}_{77}=-\left(1-\frac{\dot{\mathrm{h}}}{2}\right) \mathrm{h}^{2} \mathrm{P}_{24}-\left(1-\frac{\dot{\mathrm{h}}}{2}\right) \mathrm{h}^{2} \mathrm{P}_{24}^{\mathrm{T}}-\frac{48(2-\dot{\mathrm{h}})}{\mathrm{h}_{\mathrm{M}}} \mathrm{Q}_{3}-8\left(1-\frac{\dot{\mathrm{h}}}{2}\right) \mathrm{hQ}_{5}-36(2-\dot{\mathrm{h}}) \mathrm{Q}_{7}, \\
& \mathrm{E}_{78}=-(1-\dot{\mathrm{h}}) \mathrm{h}^{2} \mathrm{P}_{25}-\left(1-\frac{\dot{\mathrm{h}}}{2}\right) \mathrm{h}^{2} \mathrm{P}_{34}^{\mathrm{T}} \\
& \mathrm{E}_{79}=-\left(1-\frac{\dot{\mathrm{h}}}{2}\right) \mathrm{h}^{3} \mathrm{P}_{44}+24\left(1-\frac{\dot{\mathrm{h}}}{2}\right) \mathrm{hQ}_{5}+192(2-\dot{\mathrm{h}}) \mathrm{Q}_{7} \\
& E_{710}=-\left(1-\frac{\dot{h}}{2}\right) h^{3} \mathrm{P}_{45} \\
& E_{88}=-(1-\dot{h}) h^{2} P_{35}-(1-\dot{h}) h^{2} P_{35}^{T}-\frac{12(1-\dot{h})}{h_{M}} Q_{4}-4(1-\dot{h}) h Q_{6}-18(1-\dot{h}) Q_{8}, \\
& \mathrm{E}_{89}=-(1-\dot{\mathrm{h}}) \mathrm{h}^{3} \mathrm{P}_{45}^{\top} \text {, } \\
& \mathrm{E}_{810}=-(1-\dot{\mathrm{h}}) \mathrm{h}^{3} \mathrm{P}_{55}+6(1-\dot{\mathrm{h}}) \mathrm{hQ}_{6}+48(1-\dot{\mathrm{h}}) \mathrm{Q}_{8} \text {, } \\
& \mathrm{E}_{99}=-96\left(1-\frac{\dot{\mathrm{h}}}{2}\right) \mathrm{h}_{5}-1152(2-\dot{\mathrm{h}}) \mathrm{Q}_{7} \text {, } \\
& E_{1010}=-12(1-\dot{h}) h Q_{6}-144(1-\dot{h}) Q_{8} \text {, } \\
& \mathrm{L}=\left[\begin{array}{lllllllllll}
\mathrm{L}_{1}^{\top} & 0 & 0 & \mathrm{~L}_{2}^{\top} & 0 & 0 & 0 & 0 & 0 & 0 & 0
\end{array}\right]^{\top}, \\
& \mathrm{X}=\left[\begin{array}{lllllllllll}
\mathrm{A} & 0 & -\mathrm{MH} & -\mathrm{I} & \mathrm{B} & 0 & 0 & 0 & 0 & 0 & \mathrm{~N}
\end{array}\right],
\end{aligned}
$$

and the other $E_{i j}=0(i, j=1,2, \ldots, 10)$. 
Proof. First, an LKF is constructed for

$$
V(x(t))=\sum_{i=1}^{5} V_{i}(x(t)),
$$

where

$$
\begin{aligned}
& V_{1}(x(t))=\xi_{1}^{T}(t) P \xi_{1}(t)+2 \sum_{i=1}^{m} \int_{0}^{c_{i}^{T} e} \lambda_{i} \varphi_{i}(s) d s, \\
& V_{2}(x(t))=\int_{t-\frac{h(t)}{2}}^{t} e^{T}(s) Q_{1} e(s) d s+\int_{t-h(t)}^{t} e^{T}(s) Q_{2} e(s) d s, \\
& V_{3}(x(t))=\int_{-\frac{h(t)}{2}}^{0} \int_{t+\theta}^{t} \dot{e}^{T}(s) Q_{3} \dot{e}(s) d s d \theta+\int_{-h(t)}^{0} \int_{t+\theta}^{t} \dot{e}^{T}(s) Q_{4} \dot{e}(s) d s d \theta, \\
& V_{4}(x(t))=\int_{-\frac{h(t)}{2}}^{0} \int_{t+\theta}^{t} e^{T}(s) Q_{5} e(s) d s d \theta+\int_{-h(t)}^{0} \int_{t+\theta}^{t} e^{T}(s) Q_{6} e(s) d s d \theta, \\
& V_{5}(x(t))=\int_{-\frac{h(t)}{2}}^{0} \int_{t+\theta}^{t}(s-t-\theta) \dot{e}^{T}(s) Q_{7} \dot{e}(s) d s d \theta+\int_{-h(t)}^{0} \int_{t+\theta}^{t}(s-t-\theta) \dot{e}^{T}(s) Q_{8} \dot{e}(s) d s d \theta,
\end{aligned}
$$

and define

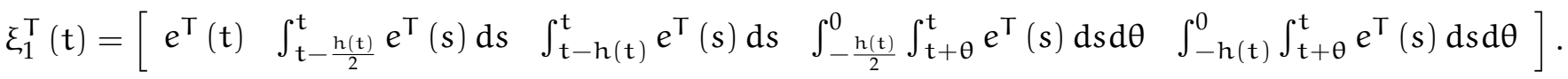

The time-derivative of function $V_{i}(x(t))$ can be calculated respectively based on the trajectory of the system (2.6) as follows:

$$
\begin{aligned}
& \dot{V}_{1}(x(t))=2 \xi_{1}^{\top}(t) P \dot{\xi}_{1}(t)+2 \varphi^{\top}\left(C^{\top} e\right) C^{\top} \wedge \dot{e}(t), \\
& \dot{V}_{2}(x(t))=e^{T}(t) Q_{1} e(t)-\left(1-\frac{\dot{h}(t)}{2}\right) e^{T}\left(t-\frac{h(t)}{2}\right) Q_{1} e\left(t-\frac{h(t)}{2}\right) \\
& +e^{T}(t) Q_{2} e(t)-(1-\dot{h}(t)) e^{T}(t-h(t)) Q_{2} e(t-h(t)), \\
& \dot{V}_{3}(x(t))=\frac{h(t)}{2} \dot{e}^{T}(t) Q_{3} \dot{e}(t)-\left(1-\frac{\dot{h}(t)}{2}\right) \int_{t-\frac{h(t)}{2}}^{t} \dot{e}^{T}(s) Q_{3} \dot{e}(s) d s \\
& +h(t) \dot{e}^{\top}(t) Q_{4} \dot{e}(t)-(1-\dot{h}(t)) \int_{t-h(t)}^{t} \dot{e}^{\top}(s) Q_{4} \dot{e}(s) d s, \\
& \dot{V}_{4}(x(t))=\frac{h(t)}{2} e^{T}(t) Q_{5} e(t)-\left(1-\frac{\dot{h}(t)}{2}\right) \int_{t-\frac{h(t)}{2}}^{t} e^{T}(s) Q_{5} e(s) d s \\
& +h(t) e^{T}(t) Q_{6} e(t)-(1-\dot{h}(t)) \int_{t-h(t)}^{t} e^{T}(s) Q_{6} e(s) d s, \\
& \dot{V}_{5}(x(t))=\frac{1}{8} h^{2}(t) \dot{e}^{\top}(t) Q_{7} \dot{e}(t)-\left(1-\frac{\dot{h}(t)}{2}\right) \int_{-\frac{h(t)}{2}}^{0} \int_{t+\theta}^{t} \dot{e}^{\top}(s) Q_{7} \dot{e}(s) d s d \theta \\
& +\frac{1}{2} h^{2}(t) \dot{e}^{T}(t) Q_{8} \dot{e}(t)-(1-\dot{h}(t)) \int_{-h(t)}^{0} \int_{t+\theta}^{t} \dot{e}^{T}(s) Q_{8} \dot{e}(s) d s d \theta .
\end{aligned}
$$

Using Lemma 2.1 to obtain

$$
-\int_{t-\frac{h(t)}{2}}^{t} \dot{e}^{\top}(s) Q_{3} \dot{e}(s) d s \leqslant-\frac{2}{h(t)} R_{1}^{\top} Q_{3} R_{1}-\frac{6}{h(t)} R_{2}^{\top} Q_{3} R_{2} \leqslant-\frac{2}{h_{M}} R_{1}^{\top} Q_{3} R_{1}-\frac{6}{h_{M}} R_{2}^{\top} Q_{3} R_{2},
$$




$$
\begin{aligned}
-\int_{t-h(t)}^{t} \dot{e}^{\top}(s) Q_{4} \dot{e}(s) d s \leqslant & -\frac{1}{h(t)} R_{3}^{T} Q_{4} R_{3}-\frac{3}{h(t)} R_{4}^{T} Q_{4} R_{4} \leqslant-\frac{1}{h_{M}} R_{3}^{T} Q_{4} R_{3}-\frac{3}{h_{M}} R_{4}^{T} Q_{4} R_{4}, \\
-\int_{t-\frac{h(t)}{2}}^{t} e^{T}(s) Q_{5} e(s) d s \leqslant & -\frac{2}{h(t)}\left(\int_{t-\frac{h(t)}{2}}^{t} e(s) d s\right)^{T} Q_{5}\left(\int_{t-\frac{h(t)}{2}}^{t} e(s) d s\right) \\
& -\frac{6}{h(t)}\left(\int_{t-\frac{h(t)}{2}}^{t} e(s) d s-\frac{4}{h(t)} \int_{t-\frac{h(t)}{2}}^{t} \int_{t-\frac{h(t)}{2}}^{s} e(s) d r d s\right)^{\top} \\
& \times Q_{5}\left(\int_{t-\frac{h(t)}{2}}^{t} e(s) d s-\frac{4}{h(t)} \int_{t-\frac{h(t)}{2}}^{t} \int_{t-\frac{h(t)}{2}}^{s} e(s) d r d s\right) \\
= & -\frac{2}{h(t)}\left(\int_{t-\frac{h(t)}{2}}^{t} e(s) d s\right)^{T} Q_{5}\left(\int_{t-\frac{h(t)}{2}}^{t} e(s) d s\right)-\frac{6}{h(t)} R_{5}^{T} Q_{5} R_{5}, \\
-\int_{t-h(t)}^{t} e^{T}(s) Q_{6} e(s) d s \leqslant & -\frac{1}{h(t)}\left(\int_{t-h(t)}^{t} e(s) d s\right)^{T} Q_{6}\left(\int_{t-h(t)}^{t} e(s) d s\right) \\
& -\frac{3}{h(t)}\left(\int_{t-h(t)}^{t} e(s) d s-\int_{t-h(t)}^{t} \int_{t-h(t)}^{s} e(s) d r d s\right)^{T} \\
& \times Q_{6}\left(\int_{t-h(t)}^{t} e(s) d s-\int_{t-h(t)}^{t} \int_{t-h(t)}^{s} e(s) d r d s\right) \\
= & -\frac{1}{h(t)}\left(\int_{t-h(t)}^{t} e(s) d s\right)^{T} Q_{6}\left(\int_{t-h(t)}^{t} e(s) d s\right)-\frac{3}{h(t)} R_{6}^{T} Q_{6} R_{6} .
\end{aligned}
$$

Using Lemma 2.2 we have

$$
\begin{aligned}
& -\int_{-\frac{h(t)}{2}}^{0} \int_{t+\theta}^{t} \dot{e}^{\top}(s) Q_{7} \dot{e}(s) d s d \theta \leqslant-6 R_{7}^{\top}\left(\begin{array}{ccc}
Q_{7} & Q_{7} & -4 Q_{7} \\
* & 3 Q_{7} & -8 Q_{7} \\
* & * & 24 Q_{7}
\end{array}\right) R_{7}, \\
& -\int_{-h(t)}^{0} \int_{t+\theta}^{t} \dot{e}^{\top}(s) Q_{8} \dot{e}(s) d s d \theta \leqslant-6 R_{8}^{\top}\left(\begin{array}{ccc}
Q_{8} & Q_{8} & -4 Q_{8} \\
* & 3 Q_{8} & -8 Q_{8} \\
* & * & 24 Q_{8}
\end{array}\right) R_{8},
\end{aligned}
$$

where

$$
\begin{aligned}
& R_{1}=e(t)-e\left(t-\frac{h(t)}{2}\right), \\
& R_{2}=e(t)+e\left(t-\frac{h(t)}{2}\right)-\frac{4}{h(t)} \int_{t-\frac{h(t)}{2}}^{t} e(s) d s, \\
& R_{3}=e(t)-e(t-h(t)), \\
& R_{4}=e(t)+e(t-h(t))-\frac{2}{h(t)} \int_{t-h(t)}^{t} e(s) d s, \\
& R_{5}=-\int_{t-\frac{h(t)}{2}}^{t} e(s) d s+\frac{4}{h(t)} \int_{-\frac{h(t)}{2}}^{0} \int_{t+\theta}^{t} e(s) d s d \theta, \\
& R_{6}=-\int_{t-h(t)}^{t} e(s) d s+\frac{2}{h(t)} \int_{-h(t)}^{0} \int_{t+\theta}^{t} e(s) d s d \theta,
\end{aligned}
$$




$$
\begin{aligned}
& R_{7}=\left(e^{\top}(t) \quad \frac{2}{h(t)} \int_{t-\frac{h(t)}{2}}^{t} e^{T}(s) d s \quad \frac{4}{h^{2}(t)} \int_{-\frac{h(t)}{2}}^{0} \int_{t+\theta}^{t} e^{T}(s) d s d \theta\right)^{\top} \text {, } \\
& R_{8}=\left(e^{T}(t) \frac{1}{h(t)} \int_{t-h(t)}^{t} e^{T}(s) d s \frac{1}{h^{2}(t)} \int_{-h(t)}^{0} \int_{t+\theta}^{t} e^{T}(s) d s d \theta\right)^{T} .
\end{aligned}
$$

At the same time, we can get the following inequalities from formula (2.2) and (2.5) for any positive diagonal matrices $T_{i}=\operatorname{diag}\left\{t_{i 1}, t_{i 2}, \ldots, t_{i m}\right\}>0,(i=1,2)$,

$$
\begin{aligned}
& -2 \eta^{\top} \mathrm{T}_{1} \eta+2 k e^{\top} C^{\top} \mathrm{T}_{1} \eta \geqslant 0, \\
& -2 \varphi^{\top} \mathrm{T}_{2} \varphi+2 k e^{\top} \mathrm{C}^{\top} \mathrm{T}_{2} \varphi \geqslant 0 .
\end{aligned}
$$

On the other hand, from the system (2.6), the following equation is true

$$
2\left(e^{T}(t) L_{1}+\dot{e}^{T}(t) L_{2}\right)(-\dot{e}(t)+A e(t)+B \eta(C e, y)-M H e(t-h(t))+N g(t))=0 .
$$

According to inequalities (3.2), (3.3), (3.4), (3.5), (3.6), (3.7), (3.8), (3.9), (3.10), (3.11), (3.12), (3.13), (3.14), (3.15), we get

$$
\dot{V}(x(t)) \leqslant \xi^{\top}(t) \Xi \xi(t) .
$$

Obviously, (3.1) implies $\dot{V}(x(t)) \leqslant \xi^{\top}(t) \Xi \xi(t)$, which means that the error dynamical system (2.5) and (2.6) is robustly asymptotically state. This completes the proof.

Remark 3.2. As is known to all, as the matrix $\Xi$ is linear with respect to $h(t)$ and $\dot{h}(t)$ in view of timevarying delay, one can test the condition on its vertices based on the convex optimization theory. Obviously, we take $h(t)=h$ and $\dot{h}(t)=0$ to get the maximum allowable delay bound because the delay is invariable for constant delay in Theorem 3.1.

Remark 3.3. The robustly asymptotically stability criterion Theorem 3.1 proposed in this paper is less conservative than existing results, which will be illustrated through two examples in the next section. Moreover, the main reason is to reduce the conservative by a piecewise analysis method, free weighting matrix approach and the new integral inequality in the Lyapunov functional.

The main reason of letting $L_{2}=\mu L_{1}, G=L_{1} M$ is to obtain the controller gain $M$ with the following synchronization criterion.

Corollary 3.4. The error system (2.6) satisfying the condition (2.5) is robustly asymptotically stable for given values $\mathrm{h}(\mathrm{t})$ in $\left\{0, \mathrm{~h}_{\mathrm{M}}\right\}, \dot{\mathrm{h}}(\mathrm{t})$ in $\left\{0, \mathrm{~h}_{\mathrm{d}}\right\}$ and $\mathrm{q}>0$, if there exist appropriate dimensional matrices $\mathrm{L}_{1}, \mathrm{G}$, and positive diagonal matrices $\Lambda=\operatorname{diag}\left\{\lambda_{1}, \lambda_{2}, \ldots \lambda_{m}\right\}>0, T_{i}=\operatorname{diag}\left\{t_{i 1}, t_{i 2}, \ldots, t_{i m}\right\}>0,(i=1,2)$, and positive matrices $\mathrm{P}=\left[\mathrm{P}_{\mathrm{ij}}\right]_{5 \times 5}, \mathrm{Q}_{\mathrm{i}}(\mathrm{i}=1,2, \ldots, 8)$ such that:

$$
\tilde{\Xi}=\left[\begin{array}{cc}
E & 0 \\
0 & -q I
\end{array}\right]+\tilde{L} \tilde{X}+\tilde{X}^{\top} \tilde{L}^{\top}+q \tau^{\top} \tau<0
$$

where

$$
\begin{aligned}
\tilde{\mathrm{L}} & =\left[\begin{array}{lllllllllll}
\mathrm{L}_{1}^{\top} & 0 & 0 & \mu \mathrm{L}_{1}^{\top} & 0 & 0 & 0 & 0 & 0 & 0 & 0
\end{array}\right]^{\top}, \\
\tilde{\mathrm{X}} & =\left[\begin{array}{lllllllllll}
A & 0 & -\mathrm{L}_{1}^{-1} \mathrm{GH} & -\mathrm{I} & \mathrm{B} & 0 & 0 & 0 & 0 & 0 & \mathrm{~N}
\end{array}\right] .
\end{aligned}
$$

Moreover, a delay feedback controller gain matrix is given by $\mathrm{M}=\mathrm{L}_{1}^{-1} \mathrm{G}$.

Remark 3.5. As a special case, when $\Delta \mathrm{A}=\Delta \mathrm{B}=0$, system (2.4) can be reduced to the following nominal form system without uncertainties:

$$
\dot{e}(\mathrm{t})=\mathrm{Ae}(\mathrm{t})+\mathrm{B \eta}(\mathrm{Ce}(\mathrm{t}), \mathrm{y}(\mathrm{t}))-\mathrm{MHe}(\mathrm{t}-\mathrm{h}(\mathrm{t})) .
$$

With Theorem 3.1 and Corollary 3.4 similar approach, we give the following globally asymptotically stability criteria for the master-slave synchronization system without uncertainties. 
Corollary 3.6. The error system (3.16) satisfying the condition (2.5) is globally asymptotically stable for given values $\mathrm{h}(\mathrm{t})$ in $\left\{0, \mathrm{~h}_{\mathrm{M}}\right\}$ and $\dot{\mathrm{h}}(\mathrm{t})$ in $\left\{0, \mathrm{~h}_{\mathrm{d}}\right\}$, if there exist appropriate dimensional matrices $\mathrm{L}_{1}, \mathrm{G}$, and positive diagonal matrices $\Lambda=\operatorname{diag}\left\{\lambda_{1}, \lambda_{2}, \ldots \lambda_{m}\right\}>0, T_{i}=\operatorname{diag}\left\{t_{i 1}, t_{i 2}, \ldots, t_{i m}\right\}>0, \quad(i=1,2)$, and positive matrices $P=\left[P_{i j}\right]_{5 \times 5}, Q_{i}(i=1,2, \ldots, 8)$ such that

$$
\hat{\Xi}=\mathrm{E}+\hat{\mathrm{L}} \hat{X}+\hat{X}^{\top} \hat{L}^{\top}<0,
$$

where

$$
\begin{aligned}
& \hat{\mathrm{L}}=\left[\begin{array}{llllllllll}
\mathrm{L}_{1}^{\top} & 0 & 0 & \mu \mathrm{L}_{1}^{\top} & 0 & 0 & 0 & 0 & 0 & 0
\end{array}\right]^{\top}, \\
& \hat{\mathrm{X}}=\left[\begin{array}{llllllllll}
A & 0 & -\mathrm{L}_{1}^{-1} \mathrm{GH} & -\mathrm{I} & \mathrm{B} & 0 & 0 & 0 & 0 & 0
\end{array}\right] .
\end{aligned}
$$

Moreover, a delay feedback controller gain matrix is given by $\mathrm{M}=\mathrm{L}_{1}^{-1} \mathrm{G}$.

\section{Examples}

In this section, we consider some numerical examples to illustrate the less conservativeness of the proposed stability criteria.

Example 4.1. Consider the following Chua's circuit

$$
\left\{\begin{array}{l}
\dot{x}=\alpha(y-h(x)) \\
\dot{y}=x-y+z \\
\dot{z}=-\beta y
\end{array}\right.
$$

with nonlinear characteristic $h(x)=m_{1} x+\frac{1}{2}\left(m_{0}-m_{1}\right)(|x+c|-|x-c|)$, and parameters $m_{0}=-1 / 7$, $\mathrm{m}_{1}=2 / 7, \alpha=9, \beta=14.28$, and $\mathrm{c}=1$. The system can be represented in Lur'e form by Yalcin et al. [21] with

$$
A=\left[\begin{array}{ccc}
-\alpha m_{1} & \alpha & 0 \\
1 & -1 & 1 \\
0 & -\beta & 0
\end{array}\right], \quad B=\left[\begin{array}{c}
-\alpha\left(m_{0}-m_{1}\right) \\
0 \\
0
\end{array}\right], \quad C=H=\left[\begin{array}{lll}
1 & 0 & 0
\end{array}\right],
$$

and $\varphi(\varepsilon)=\frac{1}{2}(|\varepsilon+1|-|\varepsilon-1|)$ belonging to the sector $[0, k]$ with $k=1$. It is worth mentioning that we take $\mu_{3}=\mu_{7}=\mu_{8}=0$ to reduce complexity factor of this example. In order to show the improvement of this paper, we summarize some comparisons in Table 1. Applying Matlab LMI-toolbox and the Remark 3.2 into the inequality (3.17), one can clearly see that the criterion in this paper provides a much less conservative result from Table 1 and Figure 1 with $h=0.227$, initial condition $x(0)=[-0.2 ;-0.3 ; 0.2], y(0)=[0.5 ; 0.1 ;-0.6]$.

What's more, the error system (3.16) with the gain matrix $M=\left[\begin{array}{lll}3.2379 & 0.5343 & -2.9219\end{array}\right]^{\top}$ is simulated and the synchronization is observed until $h \leqslant 0.295$. No feasible point is found for $h>0.295$. The simulation result with $h=0.295$ is shown in Figure 2. While the synchronization can be manifested until the maximum delay $h=0.28$ in [10], $h=0.213$ in [21] and [7], $h=0.222$ in [20], $h>0.25$ in [8] respectively. This implies that our criterion is less conservative than that in [3] and [7, 8, 10, 20, 21].

Example 4.2. Consider the following class of uncertainty Lur'e system with time-varying delay:

$$
\dot{e}(\mathrm{t})=(\mathrm{A}+\Delta \mathrm{A}(\mathrm{t})) e(\mathrm{t})+(\mathrm{B}+\Delta \mathrm{B}(\mathrm{t})) \eta(\mathrm{Ce}(\mathrm{t}), \mathrm{y}(\mathrm{t}))-\mathrm{MHe}(\mathrm{t}-\mathrm{h}(\mathrm{t})),
$$

where

$$
\begin{gathered}
A=\left[\begin{array}{ccc}
-7.2 & 1.6 & 0.8 \\
1 & -5.8 & 0 \\
3 & 11.25 & -4
\end{array}\right], \quad B=\left[\begin{array}{l}
3.7 \\
5.6 \\
4.1
\end{array}\right], \quad C=H=\left[\begin{array}{lll}
1 & 0 & 0
\end{array}\right], \\
E_{a}=\left[\begin{array}{ccc}
0.1 & 0 & 0 \\
0 & 0.1 & 0 \\
0 & 0 & 0.1
\end{array}\right], \quad E_{b}=\left[\begin{array}{c}
0.1 \\
0 \\
0
\end{array}\right], \quad N=\left[\begin{array}{lll}
1 & 0 & 0 \\
0 & 1 & 0 \\
0 & 0 & 1
\end{array}\right] .
\end{gathered}
$$


Table 1: Maximum allowed delay $h_{M}$ for Chua circuits with $\mu=0.3$.

\begin{tabular}{ccc}
\hline & Maximum allowed $h_{M}$ & Control gain $M$ \\
\hline Yalcin et al. [21] & 0.039 & {$[6.0229 ; 1.3367 ;-2.1264]$} \\
Xiang et al. [20] & 0.120 & {$[6.2121 ; 1.0868 ;-6.0359]$} \\
Han [7] & 0.141 & {$[6.0229 ; 1.3367 ;-2.1264]$} \\
He et al. [8] & 0.180 & {$[3.9125 ; 0.9545 ;-3.8273]$} \\
Li et al. [10] & 0.183 & {$[4.1455 ; 0.9250 ;-4.2596]$} \\
Ge et al. [5] & 0.185 & {$[4.0779 ; 0.9087 ;-4.3430]$} \\
Corollary 3.6 & 0.227 & {$[3.2379 ; 0.5343 ;-2.9219]$} \\
\hline
\end{tabular}

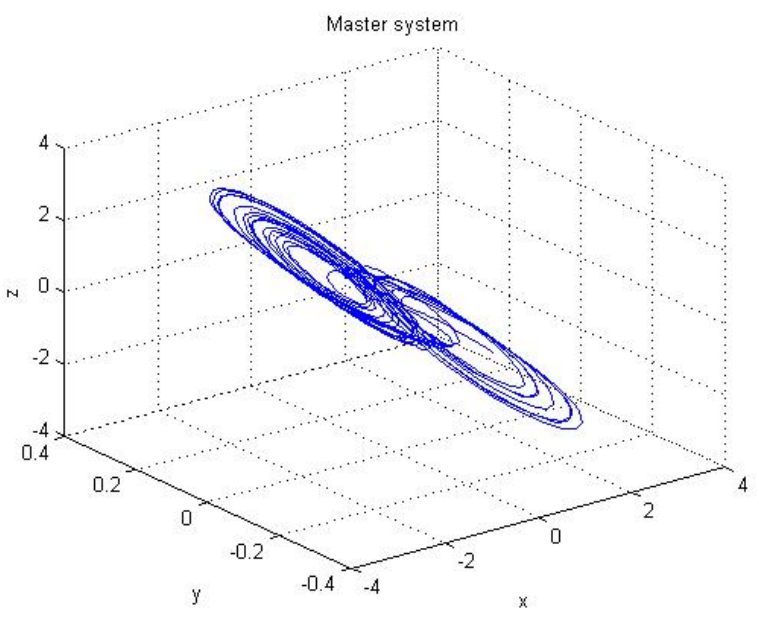

(a) Master system

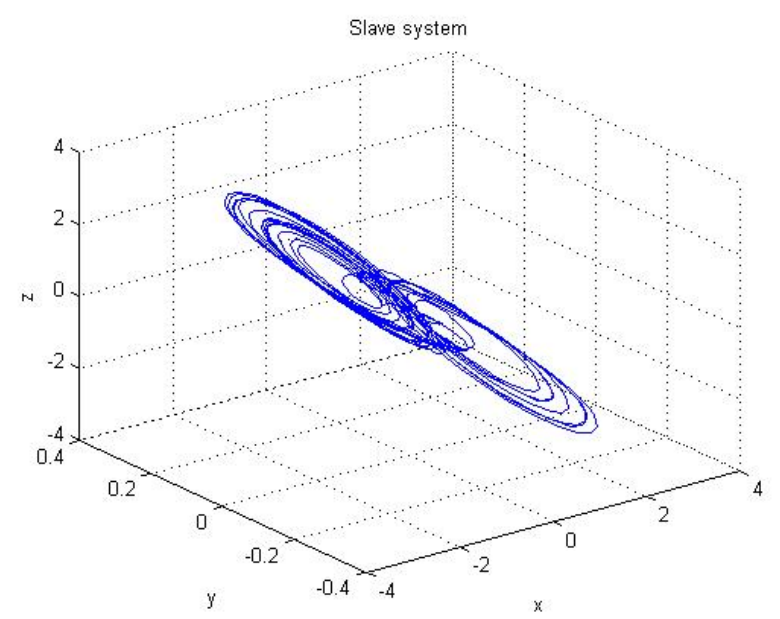

(b) Slave system

Figure 1: The trajectory of master-slave system with $h=0.227$.

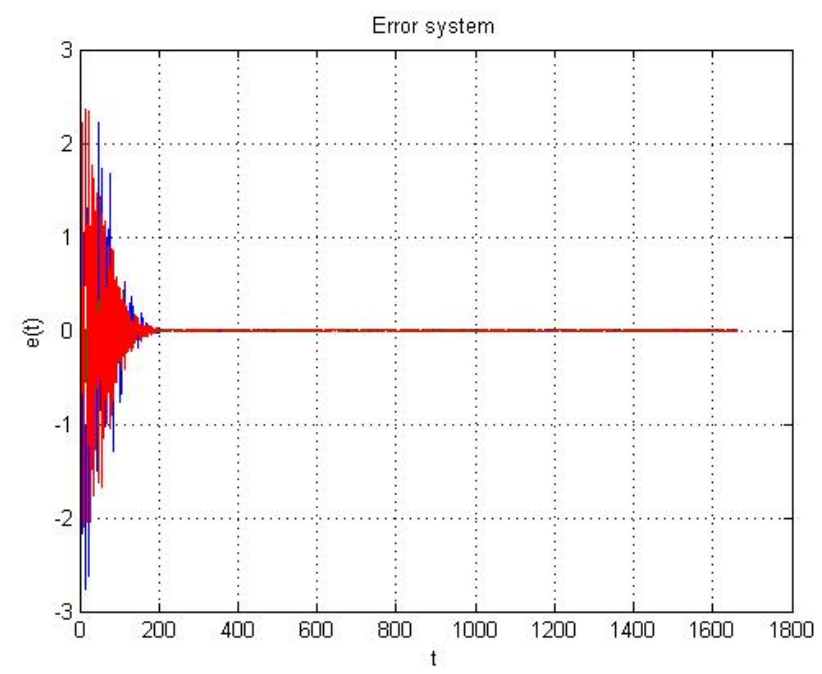

Figure 2: Simulation results for master-slave synchronization with $h=0.295$. 
Table 2: Maximum allowed delay $h_{M}$ for system (4.1) with $\mu=0.3$.

\begin{tabular}{ccc}
\hline$h_{\mathrm{d}}$ & Maximum allowed $h_{M}$ & Control gain $M$ \\
\hline 0 & 0.412 & {$[1.4957 ; 0.5247 ; 7.0373]$} \\
0.1 & 0.391 & {$[1.4410 ; 0.5543 ; 7.1561]$} \\
0.3 & 0.349 & {$[1.3301 ; 0.6137 ; 7.3543]$} \\
0.5 & 0.302 & {$[1.2957 ; 0.6870 ; 7.1822]$} \\
0.7 & 0.246 & {$[1.2495 ; 0.7116 ; 7.2897]$} \\
0.9 & 0.159 & {$[1.2191 ; 0.7574 ; 7.2088]$} \\
\hline
\end{tabular}

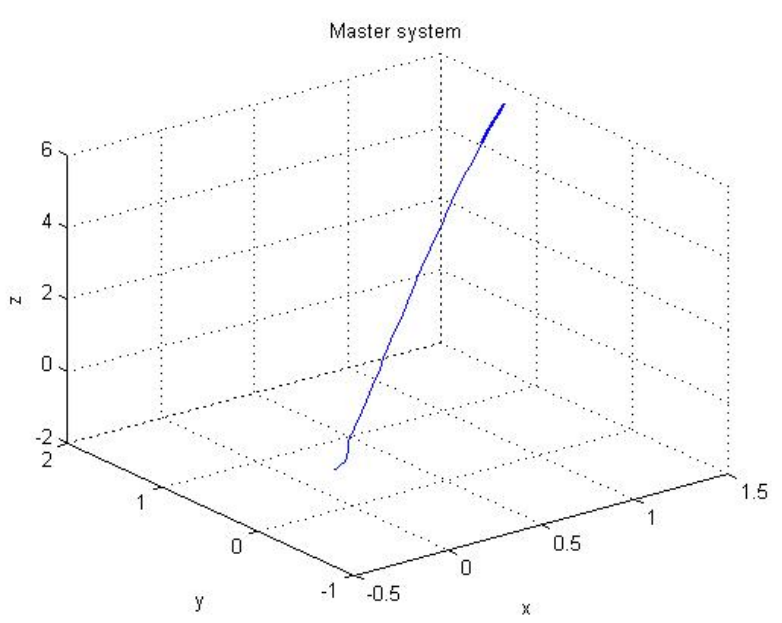

(a) Master system

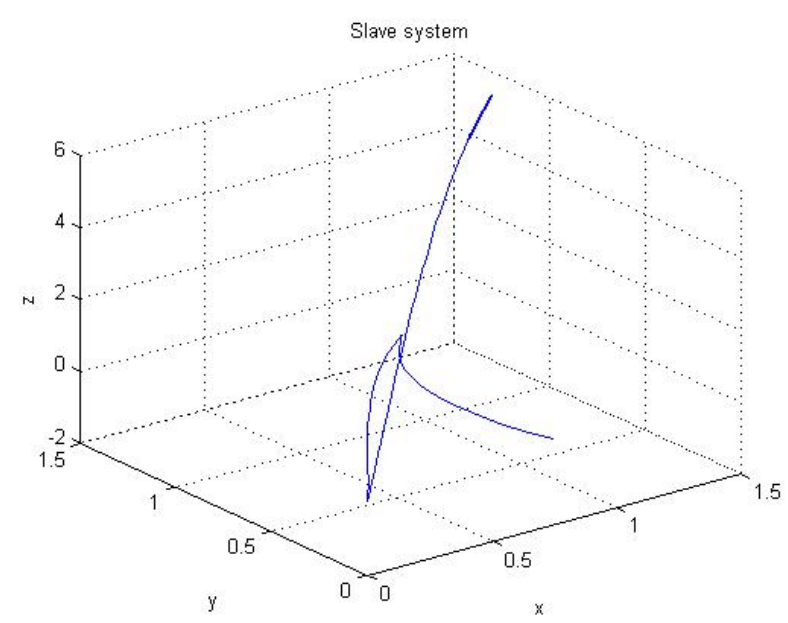

(b) Slave system

Figure 3: The trajectory of master-slave system with $h=0.412$.

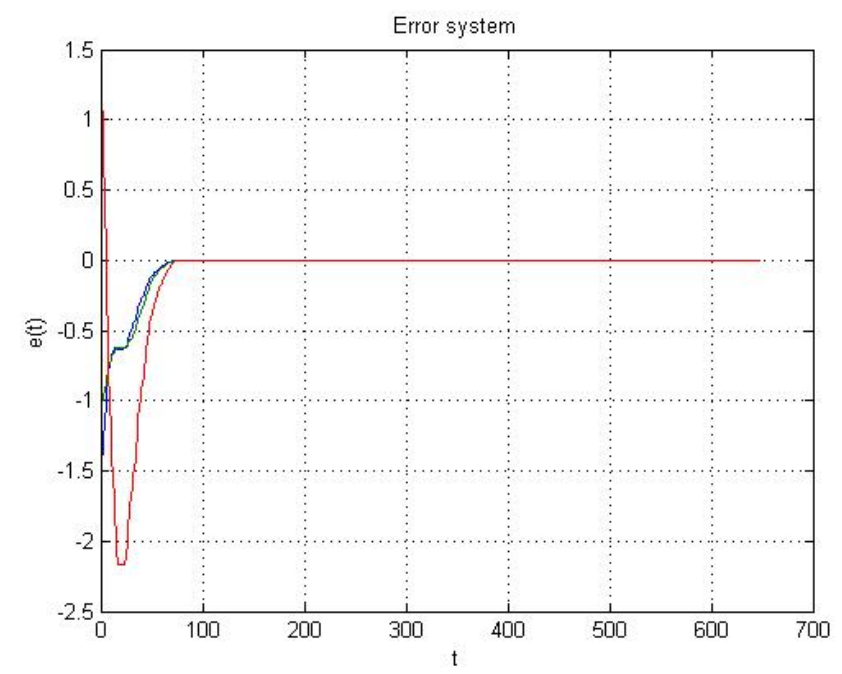

Figure 4: Simulation results for master-slave synchronization with $\mathrm{h}=0.412$.

For Example 4.2, our results of the maximum allowable delay hound $h_{M}$ and control gain $M$ for different values of $h_{d}$ are listed in Table 2 based on the Remark 3.2. It is clear to show that the maximum allowable delay bound $h_{M}$ and control gain $M$ are dependent on different values of $h_{d}$. The values of 
$h_{d}$ increase as the maximum allowable delay $h_{M}$ decreases. In order to reduce the complexity of the calculation, the synchronization criteria are given as $\mu=0.3$, and the simulation results are given as $\varphi(\varepsilon)=|\sin (|\varepsilon|)|, k=1$ with initial condition $x(0)=[-0.5 ;-0.8 ; 0.7], y(0)=[0.9 ; 0.2 ;-0.4]$ in Figures 3,4 . It is clear that the state trajectories approach to zero asymptotically.

\section{Conclusion}

In this paper, we have studied the problem of master-slave synchronization for uncertain Lur'e system with time-varying delayed feedback control by using a new Lyapunov-Krasovskii functional. The LKF contains not only double-integral terms but also triple-integral terms. Using some effective techniques, such as the new integral inequality introduced firstly here, a piecewise analysis method, and the general free-weighting matrix method, some sufficient conditions on the existence of a delayed error feedback controller derived in the form of LMIs are less conservative than existing results. Moreover, we have designed the controller by solving a set of LMIs. It can be shown that the obtained conditions are less conservative than previously existing results through numerical examples.

\section{Acknowledgment}

The first author is supported by the Scientific Research Fund Project in Yunnan Province Department of Education (Grant No.2015J069), the Specialized Research Fund For The Doctoral Program of Higher Education (Grant No. 135578), the National Natural Science Foundation of China (Grant No.11461082, 11601474).

\section{References}

[1] J.-D. Cao, H. X. Li, D. W. C. Ho, Synchronization criteria of Lur'e systems with time-delay feedback control, Chaos Solitons Fractals, 23 (2005), 1285-1298. 1

[2] G.-R. Chen, X.-N. Dong, From chaos to order, Methodologies, perspectives and applications, With a foreword by Alistair Mees, World Scientific Series on Nonlinear Science, Series A: Monographs and Treatises, World Scientific Publishing Co., Inc., River Edge, NJ, (1998).

[3] P. F. Curran, L. O. Chua, Absolute stability theory and the synchronization problem, Internat. J. Bifur. Chaos Appl. Sci. Engrg., 7 (1997), 1375-1382. 1, 4.1

[4] P. F. Curran, J. A. K. Suykens, L. O. Chua, Absolute stability theory and master-slave synchronization, Internat. J. Bifur. Chaos Appl. Sci. Engrg., 7 (1997), 2891-2896. 1

[5] C. Ge, C.-C. Hua, X.-P. Guan, Master-slave synchronization criteria of Lur'e systems with time-delay feedback control, Appl. Math. Comput., 244 (2014), 895-902. 1

[6] H.-M. Guo, S.-M. Zhong, Synchronization criteria of time-delay feedback control system with sector-bounded nonlinearity, Appl. Math. Comput., 191 (2007), 550-559. 1

[7] Q.-L. Han, New delay-dependent synchronization criteria for Lur'e systems using time delay feedback control, Phys. Lett. A, 360 (2007), 563-569. 4.1, 1

[8] Y. He, G.-L. Wen, Q.-G. Wang, Delay-dependent synchronization criterion for Lure systems with delay feedback control, Internat. J. Bifur. Chaos Appl. Sci. Engrg., 16 (2006), 3087-3091. 1, 4.1, 1

[9] D. H. Ji, J. H. Park, S. C. Won, Master-slave synchronization of Lur'e systems with sector and slope restricted nonlinearities, Phys. Lett. A., 373 (2009), 1044-1050. 1

[10] T. Li, J.-J. Yu, Z. Wang, Delay-range-dependent synchronization criterion for Lure systems with delay feedback control, Commun. Nonlinear Sci. Numer. Simul., 14 (2009), 1796-1803. 4.1, 1

[11] X.-X. Liao, G.-R. Chen, Chaos synchronization of general Lure systems via time-delay feedback control, Internat. J. Bifur. Chaos Appl. Sci. Engrg., 13 (2003), 207-220. 1

[12] H. Mkaouar, O. Boubaker, Chaos synchronization for master slave piecewise linear systems: application to Chua's circuit, Commun. Nonlinear Sci. Numer. Simul., 17 (2012), 1292-1302. 1

[13] A. Seuret, F. Gouaisbaut, Integral inequality for time-varying delay systems, European Control Conference (ECC 2013), Zurich, Switzerland, (2013), 6 pages. 2.1

[14] A. Seuret, F. Gouaisbaut, Integral inequality for time-varying delay systems, Automatica., 49 (2013), 2860-2866. 2.1

[15] Q.-K. Shen, T.-P. Zhan, A novel adaptive synchronization control of a class of master-slave large-scale systems with unknown channel time-delay, Commun. Nonlinear Sci. Numer. Simul., 22 (2015), 83-91. 1

[16] J. Sun, G. P. Liu, J. Chen, Delay-dependent stability and stabilization of neutral time-delay systems, Internat. J. Robust Nonlinear Control, 19 (2009), 1364-1375. 2.4 
[17] J. A. K. Suykens, P. F. Curran, L. O. Chua, Robust synthesis for master-slave synchronization of Lur'e systems, IEEE Trans. Circuits Syst. I: Fundam. Theor. Appl., 46 (1999) 841-850. 1

[18] J. A. K. Suykens, J. Vandewalle, Master-slave synchronization of Lur'e systems, Internat. J. Bifur. Chaos Appl. Sci. Engrg., 7 (1997), 665-669. 1

[19] J.-W. Xia, J. H. Park, H.-B. Zeng, Improved delay-dependent robust stability analysis for neutral-type uncertain neural networks with Markovian jumping parameters and time-varying delays, Neurocomputing, 149 (2015), 1198-1205. 1

[20] J. Xiang, Y.-J. Li, W. Wei, An improved condition for masterslave synchronization of Lur'e systems with time delay, Phys. Lett. A, 362 (2007), 154-158. 1, 4.1, 1

[21] M. E. Yalcin, J. Suykens and J. Vandewalle, Masterslave synchronization of Lur'e systems with time-delay, Internat. J. Bifur. Chaos Appl. Sci. Engrg., 11 (2001), 1707-1722. 1, 4.1, 1

[22] X.-M. Zhang, M. Wu, J.-H. She, Y. He, Delay-dependent stabilization of linear systems with time-varying state and input delays, Automatica J. IFAC, 41 (2005), 1405-1412. 1 